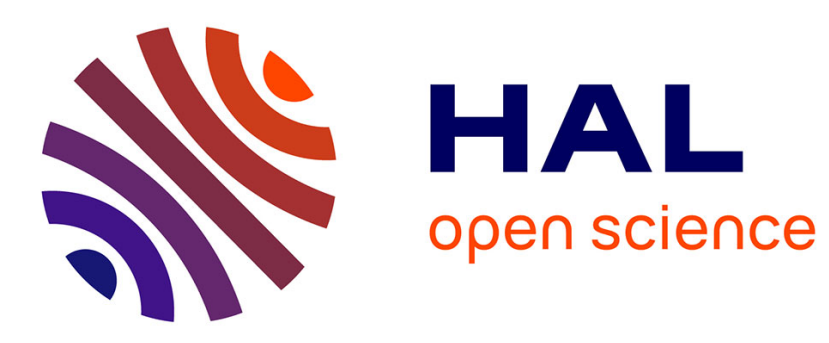

\title{
La mer et l'approvisionnement de la ville de Rome
}

Catherine Virlouvet

\section{To cite this version:}

Catherine Virlouvet. La mer et l'approvisionnement de la ville de Rome. Pascal Arnaud; Philip de Souza; Christian Buchet. The Sea in History. The Ancient World / La Mer dans l'Histoire. L'Antiquité, Boydell and Brewer, pp.268-282, 2016, 978178327157 3. hal-03539551

\section{HAL Id: hal-03539551 \\ https://hal.science/hal-03539551}

Submitted on 31 Jan 2022

HAL is a multi-disciplinary open access archive for the deposit and dissemination of scientific research documents, whether they are published or not. The documents may come from teaching and research institutions in France or abroad, or from public or private research centers.
L'archive ouverte pluridisciplinaire HAL, est destinée au dépôt et à la diffusion de documents scientifiques de niveau recherche, publiés ou non, émanant des établissements d'enseignement et de recherche français ou étrangers, des laboratoires publics ou privés. 


\section{La mer et l'approvisionnement de la ville de Rome.}

Catherine Virlouvet

Dans les derniers siècles de la République Rome atteignit un nombre d'habitants qui ne lui permit plus de s'approvisionner sur son arrière-pays. On peut discuter à l'infini sur les chiffres de population, qui ne sont que des estimations. Les indices convergent cependant : la ville, devenue capitale d'un empire qui s'étendait peu à peu à l'ensemble du bassin méditerranéen, comptait plusieurs centaines de milliers d'habitants et dépassa certainement le million au début de l'Empire ${ }^{1}$. On estime aujourd'hui qu'elle garda jusqu'au Bas Empire une population hors norme pour l'époque, de plusieurs centaines de milliers d'habitants, malgré les difficultés connues par Rome à partir de la fin du IIIe siècle ${ }^{2}$.

\section{Des besoins gigantesques.}

D'autres estimations encore permettent d'appréhender, de manière imparfaite, les quantités impressionnantes de denrées alimentaires dont avait besoin l'Urbs pour pourvoir à l'alimentation de cette population en produits de base: chaque année, à peu près 420000 tonnes de céréales, au moins $150000 \mathrm{hl}$ d'huile - auxquels il faudrait ajouter l'huile italienne-, et une consommation de vin estimée entre 1,5 et 2,2 millions $\mathrm{d}^{\prime} \mathrm{hl}^{3}$.

Par ailleurs, l'Italie tout entière présentait un niveau de population urbaine supérieure à celui que pouvait supporter l'agriculture locale : on estime entre 20 et $25 \%$ le taux de population vivant dans les cités d'Italie, à une époque où l'autosuffisance n'est pas assurée dès lors que la population occupée à des activités agricoles descend en dessous de $90 \%$ environ. Le ravitaillement qui parvenait par la mer de contrées parfois fort lointaines n'était pas uniquement destiné à l'Urbs.

Rome dut donc importer les denrées qu'elle consommait. Hormis la viande en provenance d'Italie, transportée sur pied jusqu'à la ville, et les légumes cultivés dans le suburbium, les autres produits alimentaires, sans parler des matériaux de construction comme le marbre et le bois, ou des métaux, étaient transportés par voie maritime et fluviale, plus adaptée techniquement au

\footnotetext{
${ }^{1}$ Parmi une bibliographie surabondante sur la démographie de Rome et de l'Italie, on renverra à Coarelli (2000), Nicolet (2000), Lo Cascio (2001 a et b), Scheidel (2001, 2007). Même les estimations de Scheidel, plus basses, font de Rome une ville gigantesque dans les conditions techniques du temps, comptant plusieurs centaines de milliers d'habitants. C'est désormais davantage sur les conditions de vie que se porte le débat: Rome était-elle une ville mouroir comme le pense W. Scheidel, ou ses habitants bénéficiaient-ils d'une alimentation et de conditions de vie meilleures que la moyenne si l'on suit l'avis d'une école de pensée bien représentée en Italie ?

${ }^{2}$ Lo Cascio, 1999.

${ }^{3}$ Cf. Tchernia 1986, pp. 21-27 ; Virlouvet 1995 ; Tchernia 2000. Ces estimations sont fondées sur des données disparates. Pour le montant en blé parvenant à Rome (qui représente plus que la consommation car il faut compter avec les pertes pendant le transport et le stockage), on s'appuie sur le rapprochement de deux textes : la mention par Flavius Joseph (Guerre des Juifs II, 386) de la part des contributions égyptiennes en céréales, qui représenterait quatre mois de la consommation romaine au Ier siècle ; une source très tardive, l'Epitome de Caesaribus (I, 6) qui transmet le chiffre de 20 millions de modii de blé comme celui du tribut de l'Egypte sous Auguste. On extrapole également, à partir des rations données par le Traité d'agriculture de Caton l'Ancien, les consommations individuelles moyennes qui représenteraient 3 à 4 modii soit 24 à 32 litres par mois. Pour l'huile le calcul est fait à partir des fragments d'amphores accumulés sur la décharge du Monte Testaccio aux trois premiers siècles de l'Empire. Il s'agit donc d'un calcul a minima car cette décharge est formée presqu'entièrement d'amphores ayant contenu de l'huile de Bétique et dans une faible quantité d'Afrique. Il n'est pas possible d'estimer la part de la consommation qui provenait d'Italie, en particulier de Sabine, dans des contenants qui ne se sont pas conservés (outres, tonneaux, etc.). Enfin pour le vin, le raisonnement s'appuie sur un calcul de consommation moyenne établi à partir des rations journalières prévues pour les travailleurs des domaines ruraux à partir des traités d'agronomie (Caton De Agr., 57) et de quelques inscriptions.
} 
transport des pondéreux. Si les régions voisines (l'Étrurie et la Campanie) furent à l'origine les principales pourvoyeuses de blé pour Rome ${ }^{4}$ à partir du IIe siècle av. J.-C. les territoires conquis outremer contribuèrent en grande part au ravitaillement de la ville, par l'impôt, les fermes des domaines publics puis impériaux, et le commerce libre. Les principales provinces qui approvisionnaient Rome en céréales étaient la Sicile, l'Afrique et l'Égypte, mais le blé venait aussi de Gaule, d'Espagne, d'Asie Mineure ${ }^{5}$. A partir de l'époque impériale, l'huile vint d'Espagne et d'Afrique. Pour le vin, si l'Italie resta toujours une grosse productrice, elle passa du statut d'exportatrice à l'époque républicaine à celui d'importatrice de vin gaulois et espagnol dès le Ier siècle ap. J.-C., ce qui était moins dû à une crise de la production viticole italienne qu'à l'accroissement de la population et donc des besoins de la péninsule 6 .

\section{$\underline{\text { Rome et la mer. }}$}

Cicéron a repris aux penseurs grecs le topos sur le danger des cités maritimes, et vantait la position de Rome, à l'écart de la mer (la ville en était alors distante d'une vingtaine de $\mathrm{km}$ ), des dangers et des mauvaises influences qu'elle pouvait présenter, tout en étant bien reliée à elle par le Tibre. Bref une situation enviable en tout point, qui aurait manifesté qu'une volonté divine avait présidé à l'implantation de la ville et lui avait assigné le destin de dominer un jour le monde. Par la mer, Rome recevait les produits du monde entier qui remontaient le doux Tibre pour finir dans ses entrepôts.

Pourtant la réalité n'était pas aussi enchanteresse. Le Tibre a un régime qui l'apparente plus aux torrents méditerranéens, les crues de printemps et d'automne, les étiages d'été rendent sa remontée aléatoire, compliquent son entretien et celui des quais et des magasins le longeant ${ }^{8}$. Le littoral du Latium ne possède pas de port naturel de haute mer'. Ostie, située à cette époque dans un environnement lagunaire, disposait d'un port fluvial à l'embouchure du Tibre, dont la profondeur variait en fonction des périodes : le Tibre dépose beaucoup d'alluvions, ce fut le cas en particulier dans les derniers siècles de la République, au moment précisément où la ville devenait de plus en plus dépendante de la mer pour son approvisionnement. Cette limite naturelle contraignit la cité à recourir à un dispositif portuaire complexe dans les premiers siècles de sa croissance, on le verra.

Par ailleurs, il faut tenir compte des conditions de la navigation antique qui impliquaient nombre de contraintes. Cependant force est de constater l'importance prise par le commerce maritime à l'époque romaine. Durant la période hellénistique, la taille des navires de commerce et leur capacité de transport s'accrurent en même temps que les trafics s'intensifiaient. L'unification progressive de la Méditerranée sous la domination romaine est sans doute un facteur d'explication majeur de ce phénomène ${ }^{10}$. L'intervention d'un pouvoir politique fort a joué un rôle essentiel dans les progrès des échanges maritimes. À la fin de la République, au moment où la population de l'Urbs était en pleine croissance, la lutte contre la piraterie conduisit le sénat et le peuple romain à octroyer des pouvoirs militaires exceptionnels à Antonius Creticus en 74 av. J.C., puis à Pompée en 67. Après la réduction de l'Egypte en province par Octave en 30 av. J.-C., la paix s'installa sur mer. Naviguer en convoi n'était plus une nécessité pour les flottes commerciales, cela pouvait même s'avérer un mauvais calcul pour les commerçants qui auraient risqué ainsi de perdre toutes leurs cargaisons dans la même tempête, même si, à l'époque de

\footnotetext{
${ }^{4}$ Virlouvet 1985, pp. 84-88.

${ }^{5}$ Pline l'Ancien, NH 18, 63 ; 66.

${ }^{6}$ Tchernia 1986, 2006.

${ }^{7}$ Cf. Cicéron, De Republica, II, 10

${ }^{8}$ Cf. Tchernia, 2003, pp. 46-51.

${ }^{9}$ Strabon V, 3, 5 .

${ }^{10}$ Cf. Scheidel 2011, pp. 21-38; Tchernia, 2011b.
} 
Sénèque encore, les navires qui transportaient début juin le blé d'Egypte à Pouzzoles ne faisaient pas le trajet seuls ${ }^{11}$.

L'Empire vit l'apogée du navire de commerce antique : les capacités des navires de haute mer allaient de 70 à 500 tonnes de port en lourd, pour des navires à un ou deux mâts (les trois mâts étaient rares) mesurant de 20 à 50 mètres de longueur. Certains navires excédaient ces capacités : celui qui transporta l'obélisque rapporté d'Egypte sur l'ordre de Caligula jaugeait 1300 tonnes, le navire du roman homonyme de Lucien transportait 1200 tonnes de céréales ${ }^{12}$. Mais ils étaient exceptionnels, la plupart avait 300 à 400 tonnes de capacité, ce qui fit d'eux malgré tout les plus gros navires circulant en Méditerranée jusqu'au XVe siècle. Cependant leur équipement restait sommaire : cette époque ne connut pas la boussole, ni le gouvernail d'étambot. Les bateaux étaient équipés d'un gouvernail latéral placé dans une position qui l'exposait aux chocs et aux vagues, mais qui était aussi, selon les experts, bien adapté aux manœuvres des bâtiments de l'époque. Par ailleurs, intervenait aussi dans le ravitaillement de Rome une importante flottille de caboteurs de dimensions beaucoup plus modestes, de 15 à $20 \mathrm{~m}$ de longueur, jaugeant entre 10 à 20 tonnes de port en lourd pour les plus petits, de 50 à 70 pour les plus gros, ainsi que de petits navires fluviomaritimes, les caudicariae ${ }^{13}$ (fig. 1 et 2).

Étant donné le peu d'instruments à leur disposition, les routes maritimes privilégiées par les capitaines des navires de commerce étaient celles qui comportaient le plus court trajet en pleine mer sans repaire visuel. On naviguait au plus près des côtes, en choisissant les vents les plus favorables. Ceci permet d'expliquer une des routes les plus fréquemment empruntées par les navires transportant les céréales égyptiennes jusqu'en Italie. Partis d'Alexandrie, ils faisaient route vers le nord, poussés par les vents d'ouest, jusqu'aux côtes de la Lycie, puis traversaient la mer Egée vers l'ouest en profitant des vents de secteur nord et se dirigeaient vers l'Italie par une route qui passait entre le Cap Malée et la Crète, pour remonter enfin vers Rome par le canal de Sicile et le détroit de Messine ${ }^{14}$. Les temps de parcours ne sont jamais faciles à estimer car ils dépendaient fortement de la présence ou de l'absence de vents favorables: dans de bonnes conditions, Carthage était à trois jours de navigation de Rome, ce qui mettait à portée de main de la capitale les richesses alimentaires, en particulier céréalières, de la province d'Afrique ${ }^{15}$. Mais l'absence de vent pouvait clouer les navires au port pendant des semaines, l'arrivée de vents contraires sur le trajet obligeait les navires à faire relâche dans l'abri le plus proche, ou les détournait de leur route, voire provoquait leur naufrage. Le récit fameux par Luc du voyage de l'apôtre Paul sur un navire de commerce joignant Myra, en Cilicie, à Rome, illustre bien les vicissitudes des transports maritimes ${ }^{16}$. Il faut rappeler enfin que la navigation était impossible pendant la saison hivernale. De novembre à mars, c'est la période du mare clausum durant laquelle il n'y avait aucune navigation hauturière et peu de cabotage. Une telle limite renforce le caractère crucial de la période de soudure, essentielle dans toutes les agricultures d'Ancien Régime, et l'importance centrale du stockage de moyenne durée (au moins un an de réserve).

\footnotetext{
${ }^{11}$ Sénèque, Lettres à Lucilius 77, 1 ; lettre datée de 64. "C'est un spectacle qui met la Campanie en fête : toute la population de Pouzzoles est sur les môles et reconnaît au milieu d'une foule de mâts les bâtiments alexandrins à la forme des voiles...».

${ }^{12}$ Cf. Pline HN XVI, 201-202 pour le navire porte obélisque de Caligula ; pour l’Isis, Lucien, Navigium, V.

13 Boetto 2011.

${ }^{14}$ Cf entre autres Pomey 1997 pp. 11-17 à propos du voyage de Saint Paul. Les entrepôts monumentaux édifiés sur la côte de Lycie sous le règne d'Hadrien, à Myra et Patara, ont été longtemps considérés comme dédiés à la conservation provisoire des denrées (blé essentiellement) provenant d'Egypte et destinées à l'Italie.

${ }^{15}$ Sur les difficultés à fournir des temps de parcours moyens par mer, voir Andreau, Virlouvet 2002, en particulier p. 16, p. 57 et s. (S. Crogiez), p. 204 et s. (S. Pittia), p. 224 et s. (A. Bérenger). Sur l'épisode des figues de Caton, Plutarque, Cato, 27, 1.

${ }^{16}$ Actes des Apôtres, XXVII, 1-44 ; XXVIII, 1-13). Cf. Pomey 1997, pp. 10-17.
} 
Ces contraintes liées au transport maritime valent pour la navigation commerciale comme pour les autres navigations antiques. Mais il faut les avoir à l'esprit lorsque l'on apprécie la place de la mer dans l'approvisionnement de Rome et de l'Italie : indispensable au transport de pondéreux en quantité nécessaire à la forte urbanisation de cet espace, elle était aussi un des principaux facteurs de risque d'irrégularités dans l'approvisionnement, donc de crises alimentaires génératrices de tensions sociales dans l'Urbs. Cela explique les interventions répétées des politiques pour pallier les difficultés liées à ce mode de transport.

\section{Le premier dispositif portuaire de Rome ${ }^{17}$.}

Ostie, le premier port de Rome, était un port fluvial. De récentes recherches ont permis, au moyen de carottages sédimentaires, de le localiser en rive gauche de l'embouchure du Tibre, au nord-ouest de la cité $^{18}$ (fig. 4). D'après les premiers résultats, la profondeur de ce bassin entre le IVe siècle et le IIe siècle av. J.-C. se situerait à $6 \mathrm{~m}$. Si les archives sédimentaires confirment les sources antiques sur l'importance du port pour Rome du point de vue militaire et commercial à l'époque républicaine, une telle profondeur n'est pas conforme à la vision traditionnelle que l'on a d'un port incapable d'accueillir les grands navires de commerce. Mais l'entretien d'un bassin d'une telle profondeur, dans une position soumise directement aux influences marines, devait être difficile. Des crues majeures du Tibre au IIe et au Ier siècle av. J.-C. ensablèrent fortement le port qui n'aurait plus présenté alors qu'une profondeur d'environ $0,50 \mathrm{~m}$. Par ailleurs, en aval de ce bassin, une barre d'alluvions apportées par le Tibre formait un seuil marin qui variait selon les saisons et les périodes, mais demeurait quoiqu'il en soit très difficile à franchir par les plus gros navires s'il n'était pas constamment dragué. Les témoignages antiques confirment ces difficultés, tel le célèbre épisode du navire amenant à Rome la statue de Cybèle à la fin du IIIe siècle, qui s'échoua sur un banc de sable avant de pouvoir remonter le Tibre ${ }^{19}$.

Pour pallier cet ensablement, on pouvait transborder la cargaison des plus gros navires à hauteur de l'embouchure ${ }^{20}$, en pleine mer, sur des navires fluviomaritimes, les caudicariae qui pénétraient dans le Tibre pour y décharger leur contenu dans les nombreux entrepôts d'Ostie ${ }^{21}$ ou le porter directement jusqu'à Rome. La remontée s'effectuait par halage animal ou humain. Il fallait trois jours, avec les étapes nocturnes, pour parcourir la vingtaine de km qui séparaient Ostie de Rome.

Au début du IIe siècle av. J.-C., en plein essor démographique de l'Urbs, les magistrats républicains aménagèrent au pied de l'Aventin, en partie sur leur fortune personnelle, un vaste port dans la partie aval de la ville, l'emporium, y construisant quais, portiques et entrepôts. Un tel souci reflète la dépendance de plus en plus grande de la cité à l'égard de la mer pour l'acheminement des denrées nécessaires à son approvisionnement. Or c'est aussi le moment où le bassin d'Ostie s'ensabla et où l'accès au port commença à poser de sérieux problèmes aux gros navires. Le dispositif alors mis en place dans les années mêmes de construction de l'emporium reposait sur un port de haute mer, Pouzzoles, distant de Rome de $240 \mathrm{~km}$ à vol d'oiseau, situé en Campanie dans la zone des Champs flégréens ${ }^{22}$. La seconde guerre punique avait permis aux

\footnotetext{
17 Pour la situation des ports cités, voir fig. 3 .

${ }^{18}$ Cf. Goiran et alii 2012 ; 2014, pp. 389-398.

${ }^{19}$ Cf. Aurelius Victor, De Viris illustribus, 46 ; Ovide, Les Fastes, 291-310.

${ }^{20}$ C'est la situation que décrit Strabon V, 3, 5. Cf. aussi fig. 1

${ }^{21}$ Cf. Rickman 1971, p. 15-86 ; Bukowiecki et alii 2007; 2008 ; Boetto et alii à paraitre. On ne connait que peu d'entrepôts datés avec certitude de l'époque républicaine à Ostie, les phases de construction les mieux documentées vont du début de l'Empire à l'époque sévérienne. De récentes prospections menées par la SSBAR, la BSR et les universités de Southampton et Cambridge ont révélé la présence, sur la rive droite du Tibre, d'au moins trois grandes structures que leur plan semble désigner comme des entrepôts (conférence de presse du 16 avril 2014, donnée à Rome par M. Barbera, P. Germoni, S. Keay, et F. Zevi).

22 Zevi 1994, 61-68, voit l'œuvre de Scipion l'Africain et de la gens Aemilia dans ce grandiose projet.
} 
Romains de mesurer les potentialités du port pour ravitailler les troupes. En 199 av. J.-C., un portorium y fut établi et une colonie romaine y fut fondée dès 194.

Les sources littéraires, les restes archéologiques en bonne part submergés des structures portuaires (quais et entrepôts), certains documents de la pratique permettent de mesurer l'ampleur de l'activité déployée par la cité qui jouait le rôle de port de redistribution pour l'Italie et pour Rome ${ }^{23}$.

A Pouzzoles, les navires de haute mer étaient déchargés. Une partie de leur cargaison était transférée dans des caboteurs plus petits qui remontaient la côte du Latium jusqu'au port fluvial d'Ostie. Par temps favorable, la distance entre la baie de Naples et l'embouchure du Tibre peut se parcourir en une journée et demie. Une autre partie des cargaisons était stockée sur place dans des entrepôts dont on connaît mal les vestiges (en raison du phénomène de "bradisisme » que connait cette région au volcanisme actif) mais que les photographies aériennes, les prospections sous-marines et les sources écrites ${ }^{24}$ permettent de restituer comme de grands bâtiments à cellules accolées selon un modèle assez semblable à celui qui domine à Ostie et à Rome. Dans ces bâtiments, on conservait sur de courtes et moyennes durées ${ }^{25}$ les denrées alimentaires destinées pour la plupart à être réexportées vers le reste de l'Italie et Rome en particulier.

Ce n'est donc pas la totalité du blé à destination de Rome débarqué à Pouzzoles à partir de la fin du printemps qui était immédiatement transférée vers Ostie. Il remontait progressivement sur des caboteurs de plus petite taille, capables d'entrer dans les bouches du Tibre. On a parfois cherché à estimer le déploiement logistique nécessaire pour assurer le stockage à proximité de Rome de quantités de céréales permettant à la ville d'assurer la soudure jusqu'à l'arrivée des nouvelles récoltes : A. Tchernia estime que, dans les meilleures conditions possibles, en prenant les fourchettes les plus basses de consommation de céréales dans l'Urbs, il faudrait au moins 90 caboteurs jaugeant environ 150 t de port en lourd et effectuant le trajet Pouzzoles-Rome de manière incessante entre juillet et octobre pour assurer des réserves suffisantes à la capitale ${ }^{26}$.

Le moindre déficit dans le nombre des navires disponibles ou dans celui des rotations mettait l'ensemble du système en péril. Une réquisition de bateaux, comme en 39 lorsque Caligula décida de former avec ceux-ci un pont entre Pouzzoles et le Cap Misène ${ }^{27}$, de mauvaises conditions météorologiques pendant les mois d'été, pouvaient compromettre la constitution de réserves et aboutir à des situations de tension sur le marché de Rome : les réserves diminuaient, le blé se faisait rare, les prix flambaient. Les ingrédients de la disette étaient réunis. Celles qui ont donné lieu à des émeutes au sein de la population de la ville furent particulièrement nombreuses dans les derniers siècles de la République et au cours du premier siècle de l'Empire ${ }^{28}$. Elles ne s'expliquent pas toujours par une défaillance de la logistique des transports : les guerres, les mauvaises récoltes dans les zones exportatrices, les inondations ou les incendies ravageant les magasins de stockage étaient aussi des causes fréquentes de disette. Cependant la très grande dépendance vis-à-vis de la mer constituait une fragilité supplémentaire : les naufrages étaient fréquents (au début du règne de Néron les tempêtes en Méditerranée furent cause de tensions dans la ville ${ }^{29}$ ), les pirates gênaient le commerce jusque dans les années 60 av. J.-C., les blocus maritimes, tel celui imposé par Sextus Pompée dans sa lutte contre les triumvirs entre 41 et 36 av. J.-C., pouvaient affamer la

\footnotetext{
${ }^{23}$ Dubois 1907 ; Zevi 1993 ; 1994 ; Camodeca 1999 ; Virlouvet 2000.

${ }^{24}$ Cf. les deux contrats de location d'entrepôts dans les archives des Sulpicii de Pouzzoles (Camodeca 1999, TPSulp 45 et 46). Cicéron mentionne aussi (De finibus II, 84) la possession d'entrepôts à Pouzzoles comme l'exemple même de placement rentable.

${ }^{25}$ L'affaire de prêt à laquelle appartient un des contrats de location d'espace dans un grand entrepôt de Pouzzoles est conclue pour deux mois seulement (TPSulp 79)

${ }^{26}$ Pomey, Tchernia 1978, p. 240 et s.

27 Dion Cassius LIX, 17, 2

28 Virlouvet 1985.

${ }^{29}$ Tacite Ann. 15, 18, 5.
} 
ville ; et l'impossibilité de faire venir du ravitaillement pendant les mois d'hiver ne permettait pas de remédier à d'autres difficultés qui n'avaient pas été provoquées par la mer (les incendies, les inondations) mais ne pouvaient pas non plus se régler grâce à elle. Bien des difficultés recensées entre la fin de la République et le premier siècle de l'Empire se situent en hiver, preuve que les problèmes d'approvisionnement tenaient moins à des questions de soudure (qui concernent le début du printemps) que de logistique ${ }^{30}$.

\section{Les grands travaux de Portus et la stabilisation du dispositif impérial (fig. 5).}

Dès la fin de la République, on chercha des remèdes aux problèmes liés au transport maritime, par la réalisation de grands travaux pour sécuriser le trajet le long de la côté tyrrhénienne et diminuer la dépendance de Rome envers un port aussi éloigné que Pouzzoles. César projeta un canal intérieur accessible aux navires de haute mer, qui aurait relié Terracine au Tibre à la sortie de Rome ${ }^{31}$. A une centaine de $\mathrm{km}$ au sud des bouches du Tibre, Terracine était le dernier port de mer bien abrité, avant la remontée jusqu'à Ostie le long d'une côte plutôt rectiligne, sans beaucoup de protection pour les navires ${ }^{32}$. Le canal imaginé par César aurait longé la côte en traversant les marais pontins à l'assèchement desquels il avait pourvu ${ }^{33}$. C'est un canal du même genre qui reçut un début de réalisation sous Néron. Les ingénieurs Severus et Celer projetèrent un canal de $240 \mathrm{~km}$ de long, partant de Pouzzoles, plus précisément du lac Averne, et aboutissant à Ostie ${ }^{34}$. Ce projet, et celui de percement de l'isthme de Corinthe, s'ils témoignent peut-être de la « folie des grandeurs » qui habitait Néron (c'est du moins ainsi que les présentent les sources anciennes), ont pourtant une grande cohérence dans leur visée, celle de faciliter l'arrivée des navires de commerce à Rome et de diminuer les fragilités dans l'approvisionnement de la capitale dues à l'éloignement d'une partie des réserves et à la période de mare clausum.

La construction d'un port de haute mer à hauteur de Rome était également envisagée depuis de nombreuses années lorsque Claude l'entreprit en 46. C'est encore à César que l'on prête le premier projet ${ }^{35}$, non réalisé en raison de sa difficulté, selon Suétone. Claude détermina l'emplacement du Portus Augusti au nord de l'embouchure du Tibre, contre l'avis de certains de ses contemporains, le Tibre alluvionnant davantage au nord de son embouchure. Cependant l'emplacement comportait peut-être une petite anse naturelle car un bateau d'époque archaïque y a été retrouvé. Il permettait aussi de rejoindre aisément le Tibre par le creusement de canaux. Celui que l'on appelle abusivement la fossa Traiana fait en réalité partie du projet claudien. Un très vaste bassin artificiel, mesurant environ 200 ha, de $8 \mathrm{~m}$ de profondeur ${ }^{36}$, protégé par deux môles curvilignes et annoncé par un grand phare, fut implanté à cet emplacement. Le port fut terminé sous le règne de Néron, en 64, mais avant même son inauguration une tempête provoqua le naufrage de 200 navires qui s'y trouvaient abrités ${ }^{37}$. Le bassin artificiel était trop ouvert aux courants marins et s'ensablait très rapidement.

Trajan compléta l'œuvre de Claude en faisant construire entre 106 et 112 un second bassin interne mieux protégé, de forme hexagonale et d'une superficie de 32 ha, communiquant au

\footnotetext{
${ }^{30}$ Sur la claire conscience de ce problème par les anciens eux-mêmes, cf. Dion Cassius LX, 11 à propos du règne de Claude.

31 Plutarque Caesar 58, 8 ; Tacite Ann. 15, 42. Plutarque prête aussi à César un projet de percement de l'isthme de Corinthe.

${ }^{32}$ Seul le site d'Antium présente un petit promontoire. La ville possédait un port à l'époque archaïque, Néron en fit reconstruire un également.

33 Suétone, Div. Iul. 44 ; Dion Cassius, XLIV, 5.

34 Tacite Ann. 15, 42 ; Suétone, Nero 31 ; Pline NH XIV, 61.

35 Suétone, Cl. 20 ; Plutarque Caes., 58, 8. On dut discuter beaucoup au début de l'Empire autour de l'aménagement d'un semblable port, au point que le thème était un des sujets donnés à leurs élèves par les professeurs de rhétorique (Quintilien, Inst. Orat. II, 21, 18 ; III, 8, 16).

${ }^{36}$ Cf. Keay 2011, p. 2.

37 Tacite, Annales, 15, 18, 3.
} 
moyen des canaux et de la darse déjà existants avec le bassin de Claude dans lequel un canal en eau profonde permettant le cheminement des navires continua à être entretenu ${ }^{38}$. Il fit aménager d'autres canaux rejoignant le complexe de Portus au Tibre vers l'amont, mais aussi vers Ostie parallèlement à la côte (fig. 6). Portus ne prit pas la place d'Ostie, les deux ensembles formaient une unique conurbation liée par le Tibre, le premier étant sans doute tourné surtout vers l'approvisionnement de Rome tandis que le second avait un rôle de redistribution en Méditerranée de produits variés ${ }^{39}$.

Trajan entreprit aussi à partir de 107 le premier port de Centumcellae (Civitavecchia), à une cinquantaine de $\mathrm{Km}$ au nord de Rome ${ }^{40}$. Les avantages du site n'étaient pas aussi évidents que ceux de Pouzzoles. Mais le cap Linaro protège le port des alluvionnements du Tibre et les monts de la Tolfa peuvent fournir de bons amers ${ }^{41}$. Les aménagements ne sont plus visibles depuis leur destruction par les bombardements durant la seconde guerre mondiale. Ils consistaient en un grand bassin délimité par deux moles terminés chacun par un phare en forme de tour dont une seule est partiellement conservée aujourd'hui, et précédés d'une digue sur laquelle on restitue un phare $^{42}$. On manque de témoignages pour évaluer avec précision le rôle joué par Civitavecchia dans le dispositif et on a sans doute tendance à le sous-évaluer. Lorsque le mauvais temps empêchait l'accès à Portus, les bateaux abordaient sans doute là, mais le rôle du port ne devait pas se limiter à cela. Il y a un pan de l'histoire de l'approvisionnement de Rome antique par la mer qui reste encore à creuser.

L'aménagement de Portus et de Civitavecchia à la fin du premier et au début du second siècle s'accompagna de la construction de grands espaces de magasins qui renforçaient considérablement les capacités de stockage à proximité de Rome, réglant en partie les problèmes logistiques évoqués auparavant. Ces espaces furent encore agrandis à l'époque sévérienne. Même à la mauvaise saison, les denrées emmagasinées par beau temps à proximité pouvaient parvenir à Rome. La fréquence des crises attestées par les sources diminue d'ailleurs sensiblement par la suite et ce fait ne s'explique sans doute pas seulement par une moins grande abondance de témoignages écrits par la suite. Cette situation nouvelle n'entraîna pas pour autant le déclin de Pouzzoles, toujours actif à l'époque impériale, tourné vers le commerce avec la Méditerranée orientale et qui ne desservait pas seulement la ville de Rome.

Semblable dispositif se maintint au moins jusqu'au Ve siècle. A cette époque, les entrepôts étaient encore entretenus, comme on a pu le constater lors de récentes recherches à Portus, dans les entrepôts sévériens ${ }^{43}$ et dans les magasins dits de Trajan. Dans ces derniers, les vides sanitaires ménagés dans les sols de certains magasins ont été refaits avec soin en plein Ve siècle ${ }^{44}$. Les naves caudicariae mises au jour lors de l'aménagement de l'aéroport de Fiumicino sont datées des IV-Ve siècles ${ }^{45}$.

\section{$\underline{\text { La part de l'Etat }}{ }^{46}$.}

Ainsi, c'est l'intervention du pouvoir romain qui permit à la capitale de l'empire de bénéficier de l'approvisionnement nécessaire, car assurer un ravitaillement régulier par la mer supposait un monde pacifié et une lourde infrastructure matérielle qui fut prise en charge par les autorités

\footnotetext{
${ }^{38}$ Le tracé précis de ce canal reste à déterminer.

${ }^{39}$ Hypothèse d'Heinzelmann 2010, p. 8.

${ }^{40}$ Cf. Pline le Jeune Ep. 6,31, 15-17.

41 Tchernia 2003, p. 53.

${ }^{42}$ Cf. Correnti, 1990 ; Quilici 1993. Le bassin principal était bordé de magasins de stockage dont les restes sont encore visibles dans les caves des immeubles d'après-guerre.

${ }^{43}$ Keay 2011, p. 7 ; Paroli, Ricci, 2011, pp. 129-146.

${ }^{44}$ Cf. Bukowiecki et alii 2011, p. 357-359.

45 Boetto 2011.

${ }^{46}$ Cette expression est empruntée à un chapitre de Tchernia 2011, pp. 133-155.
} 
publiques. Ce trait ne doit pas pour autant laisser penser que les empereurs assumèrent l'ensemble de l'approvisionnement de la capitale dans un système « étatisé » avant la lettre. Même les infrastructures lourdes que constituaient les édifices de stockage présents dans les ports n'ont pas seulement été construites et maintenues sur initiative publique ${ }^{47}$.

La question du périmètre exact d'intervention de la préfecture de l'annone créée par Auguste au début de notre ère reste ouverte. Il est certain qu'elle passait, pour l'acheminement des denrées provenant des impôts et des fermes, des contrats avec les privés, transporteurs maritimes et propriétaires de navires. Le service rendu par ceux-ci tendit à se transformer en un munus à l'époque tardive seulement ${ }^{48}$.

Par ailleurs, au début de l'Empire, certaines décisions de l'autorité publique visèrent spécifiquement à encourager le commerce maritime des produits à destination de Rome. Tacite fait allusion aux mesures de Tibère pour remédier aux aléas du transport maritime ${ }^{49}$. Claude fit face dès 41 à de sérieuses difficultés de ravitaillement en partie causées par le fameux pont de bateaux entre Pouzzoles et le Cap Misène, dernière "folie» du règne de Caligula. En 51 de nouveau, il dut affronter l'hostilité de la foule qui se plaignait de la cherté du pain. Il prit alors des mesures incitant au commerce du blé en période de mare clausum ${ }^{50}$. Ces mesures offraient des avantages aux constructeurs de navires de plus de 10000 modii (70 tonnes environ) qui feraient le commerce pendant l'hiver. Ces décisions se maintinrent sur la longue durée : l'édit de Claude est encore cité par Suétone et les juristes des II-IIIe siècles ${ }^{51}$. Comme l'a bien montré A. Tchernia ${ }^{52}$, la petitesse du tonnage minimal nécessaire pour obtenir les avantages consentis par l'empereur ${ }^{53}$ prouve qu'un des objectifs de Claude était de faire circuler en période hivernale les caboteurs qui transportaient le blé entre Pouzzoles et Ostie. On soulignera qu'aucun des témoignages portant sur cet édit de Claude n'affirme que le blé ainsi transporté serait uniquement du blé annonaire, produit des impôts et des fermes. Suétone qualifie le blé de mercatura ${ }^{54}$ ce qui plaide pour la prise en compte des céréales transportées dans le cadre du commerce libre.

Transporteurs maritimes, propriétaires de navires, propriétaires et gestionnaires d'entrepôts, commerçants, mesureurs, porte-faix, etc. furent autant d'interlocuteurs du pouvoir romain participant à la lourde tâche d'assurer à la capitale millionnaire, par la voie maritime et fluviale essentiellement, un approvisionnement régulier et de qualité.

Pline l'Ancien exagérait à peine en disant que toutes les productions de l'univers transitaient par le Tibre vers Rome ${ }^{55}$. Les Anciens, tout comme nous, s'émerveillaient d'un semblable prodige. Il ne fait pas de doute qu'un tel degré de développement du commerce s'explique par les moyens dont disposait la puissance publique. C'est elle qui sécurisa les mers, c'est majoritairement elle qui construisit et entretint les ports et leurs infrastructures, qui fut à l'origine du transport, de la conservation et de la distribution de grandes quantités de blé annonaire. Cependant même le commerce du blé n'échappait pas entièrement à l'initiative privée, sans parler du commerce de

\footnotetext{
47 Tels les deux entrepôts de Pouzzoles connus par les tablettes des Sulpicii (cf. ci-dessus n. 22) et nombre d'entrepôts de la ville de Rome. Cf. Virlouvet 2011 ; à paraittre.

${ }^{48}$ L'inscription dite des naviculaires d'Arles trouvée à Beyrouth CIL III 14165 (8) témoigne de l'existence de semblables relations contractuelles au début du IIIe siècle, dans un contexte encore assez libre. Cf. Virlouvet 2004. $\mathrm{Au}$ IVe siècle le service du transport pour le compte de l'annone est devenu une obligation, un munus. Cf. Sirks, 1991 ; De Salvo 1992.

49 Tacite, Ann. 4, 6, 6.

50 Tacite, Ann. 12, 43, 1-2 ; Suétone Cl. XVIII, 3-4 - XIX.

${ }^{51}$ Gaius, Institutes, I, 32, c ; Digeste III, 6 (Ulpien).

52 Pomey, Tchernia 1978, p. 237 et s.

${ }^{53}$ L'exemption de la loi Papia Poppaea pour les citoyens romains, la citoyenneté pour les Latins, les prérogatives des mères de quatre enfants pour les femmes.

${ }^{54}$ Suétone Cl. XVIII 3-4-XIX (...) et naves mercaturae causa fabricantibus magna commoda constituit (...) Et il assura aux armateurs construisant des navires pour faire ce commerce de grands avantages...

55 Pline l'Ancien, NH III, IX, 54
} 
l'huile de Bétique ou du vin gaulois assurés par des privés. Il faut souligner l'hétérogénéité du commerce romain, pour l'Urbs comme pour l'ensemble de l'empire.

L'approvisionnement de la capitale reposait sur un système sophistiqué, dans lequel la mer a joué un rôle essentiel. Malgré les limites techniques du temps, ce système de "commerce imbriqué " ${ }^{56}$ fut très efficace parce que les initiatives privées s'appuyaient sur l'organisation par l'État d'une remarquable infrastructure matérielle et fonctionnelle.

56 Tchernia 2011, p. 155. 